\title{
Repliken auf den Beitrag ,Coaching durch die Führungskraft als Beratungsformat" von Wolfgang Kühl, Erich Schäfer, Andreas Lampert
}

\author{
A. Schreyögg • E. Lippmann
}

Online publiziert: 15. Februar 2019

(C) Springer Fachmedien Wiesbaden GmbH, ein Teil von Springer Nature 2019

\section{Astrid Schreyögg}

Im Gegensatz zu dem Organisationssoziologen Stephan Kühl sind Wolfgang Kühl und vermutlich auch seine Ko-Autoren Sozialpädagogen. Aus dieser Differenz der „Namensvetter“ erklärt sich vermutlich auch das Plädoyer von Wolfgang Kühl für Coaching durch Führungskräfte, während diese Position von Stephan Kühl noch nie $\mathrm{zu}$ vernehmen war und vermutlich auch in Zukunft nicht zu vernehmen sein wird.

Das Problem von Coaching durch Vorgesetzte lässt sich am ehesten an Beispielen verdeutlichen: Stellen wir uns eine hochformalisierte, d.h. stark hierarchisch gestaltete Organisation wie etwa VW vor, in der alle Abläufe durch viele formale Regeln überformt sind. In diesem System ist auch jeder Karriereschritt eines Mitarbeiters vom Votum und von der Leistungsbeurteilung seines Vorgesetzten abhängig. Dann ist es aber schwer vorstellbar, dass dieser Mitarbeiter seine Ängste, seine Bedenken, seine beruflichen oder privaten Sorgen seinem Vorgesetzten im Rahmen eines Coachings anvertrauen würde. Das wird er auch dann nicht tun, wenn dieser Vorgesetzte immer sehr wertschätzend und wohlwollend mit ihm und seinen anderen Unterstellten interagiert, wenn er also einen betont integrativen Führungsstil praktiziert. Genauso wenig werden sich Lehrkräfte im staatlichen Schulwesen, in dem Leistungsbeurteilungen durch die Schulleitung für ihre Beförderung maßgeblich sind, diesem Schulleiter mit ihren beruflichen Sorgen und Ängsten anvertrauen. Sie werden es auch dann eher nicht tun, wenn dieser Schuleiter einen ausgeprägt

\footnotetext{
Dr. A. Schreyögg $(\bowtie)$

Breisgauer Str. 29, 14129 Berlin, Deutschland

E-Mail: info@schreyoegg.de

Prof. Dr. E. Lippmann

ZHAW Zürcher Hochschule für Angewandte Wissenschaften, Pfingstweidstrasse 96, CH-8037 Zürich, Schweiz

E-Mail: eric.lippmann@zhaw.ch
} 
liebenswürdigen Führungsstil und vielleicht sogar einen regelrechten Schmusekurs gegenüber seinen Lehrkräften praktiziert.

Weniger reserviert verhalten sich Mitarbeiter gegenüber Projektleitern, wenn diese nur eine fachliche, nicht aber eine disziplinarische Vorgesetztenfunktion haben. Dann kommt der Leitung nur eine begrenzte Macht gegenüber dem Mitarbeiter zu, die sich in Empfehlungen oder informellen Anmerkungen zu seiner Leistung oder seiner sonstigen Haltung erschöpft. Machtvoller Einfluss erfolgt immer erst durch einen disziplinarischen Vorgesetzten, was dann eben auch manifeste negative Konsequenzen nach sich ziehen kann. De facto bestehen aber auch in Kontexten des Projektmanagements selten so vertrauensvolle Beziehungen zwischen Leitung und Mitarbeitern, die ein Coaching nahelegen würden. Trotz vielfältiger Entbürokratisierungsbemühungen der letzten Jahre lässt sich insgesamt behaupten, dass in weiten Teilen von Wirtschaft und Verwaltung auch heute noch die meisten Organisationen mehr oder weniger strikt hierarchisch gestaltet sind. Nur vereinzelt finden wir nichthierarchische Organisationen oder organisatorische Einheiten.

Wolfgang Kühl et al. ist allerdings durchaus zuzustimmen, wenn sie behaupten, dass sich das Führungsverständnis in vielen Organisationen heute positiv verändert hat. Und selbstverständlich ist den Autoren auch beizupflichten, dass es heute mehr denn je um maximal reflektierte Führungshaltungen bzw. -stile in Organisationen sogar notwendigerweise gehen muss, um fachlich qualifizierte Mitarbeiter in den jeweiligen Organisationen zu halten. Und es ist auch geradezu trivial zu betonen, dass es eine Aufgabe von Vorgesetzten ist, ihre unterstellten Mitarbeiter bei ihrer Aufgabenerfüllung maximal fachlich zu unterstützen. Das gehört nämlich zu der Funktion der Personalführung eines jedes Managers.

Coaching ist aber doch etwas anderes. Es ist eine vertiefte psychologische Beratung, die in ihrem Selbstverständnis und in ihren Auswirkungen eine formale Managementfunktion weit überschreitet. Sie setzt eine Vertrauensbeziehung zwischen Vorgesetzten und unterstellten Mitarbeitern voraus, die es in hierarchischen Verhältnissen immer nur begrenzt oder gar nicht geben kann.

So sitzen Wolfgang Kühl und seine Ko-Autoren einem Missverständnis auf, das für sozialpädagogische Milieus geradezu typisch ist: Wenn Vorgesetzte lieb, nett und freundlich zu ihren Mitarbeitern sind, versuchen sie vergessen zu machen, dass sie als Vorgesetzte über eine Positionsmacht verfügen, von der empfindliche Sanktionen ausgehen könnten. Dementsprechend phantasieren sie dann auch nahtlos die Möglichkeit von Coaching durch Vorgesetzte. Das gilt übrigens auch für große Teile der US-Amerikanischen Coaching-Literatur, weil man dort ebenfalls selten den organisatorischen Kontext als relevanten Systemfaktor berücksichtigt. Auf einer Makroebene entspricht das übrigens auch dem Missverständnis von Trump gegenüber Putin: Wenn man sich zu zweit freundschaftlich begegne, könne man sich gegenseitig vorbehaltlos vertrauen, und der Weltfrieden sei gesichert. 


\section{Eric Lippmann}

Es ist ja grundsätzlich erfreulich, dass die Diskussion um das Coaching durch die Führungskraft als Beratungsformat weitergeführt wird. Den Autoren kommt sicher das Verdienst zu, die aktuelle Literatur aufgearbeitet zu haben und die Diskussion im Zeitalter einer ,veränderten Organisations- und Reflexionskultur“ (Abstract) weiter zu beleben. Im Artikel werde ich ja als einer der Kritiker genannt. Auch wenn sich gemäß Tonhäuser (2010) das Coaching durch die Führungskraft in zahlreichen Unternehmen etabliert haben soll, sagt dies aber noch nichts über dessen Sinnhaftigkeit. Und es heißt auch nicht, dass dadurch meine Skepsis verschwunden ist.

In meiner Replik beginne ich deshalb mit den Organisationen, bei denen die Hierarchien fast ganz abgeschafft worden sind. Diese Organisationsformen sind in den letzten Jahren durch zahlreiche Publikationen in gewisser Weise ,in Mode“ gekommen (vgl. Laloux 2015; Robertson 2016; Oestereich und Schröder 2017). Gerade in der letztgenannten Publikation wird klar umschrieben, was die Rolle eines/r Coach in einem kollegial geführten Unternehmen sein kann: „Ein Coach bzw. Prozessbegleiter trifft selber keine inhaltlichen Entscheidungen, sondern leitet seine Klienten zur Selbstreflexion an. (...) Diese Rolle hat keine inhaltliche Entscheidungsmacht. Sie kann die Prinzipien, Regeln und andere Rollen nicht ändern" (Oestereich und Schröder 2017, S. 121). Das heißt: Selbst in Organisationen ohne Hierarchien wird darauf geachtet, dass ein/e Coach bei den Anliegen, die gecoacht werden, selber nicht (mit)entscheidet und auch keine Macht hat, an den Gegebenheiten etwas zu ändern. Doch nicht-hierarchische Organisationen finden wir - wie Schreyögg in ihrer Replik in diesem Heft richtig anmerkt - nur vereinzelt, zumindest derzeit.

Wenden wir uns also wieder der Mehrheit zu, den Führungskräften in hierarchischen Organisationen. Hier bleibt die Problematik, dass die Führungskräfte nie frei von eigenen Interessen coachen können, weiterhin bestehen. Dazu vermerken Kühl et al. in ihrem Artikel selbst, dass der „Umgang mit möglichen offenen und latenten Rollenkonflikten in vielerlei Hinsicht noch nicht hinreichend bedacht zu sein“ scheint. Wie dann diese möglichen Rollenkonflikte aus dem Weg geräumt werden sollten, damit ein professionelles Coaching durch die Führungskraft stattfinden kann, darüber lässt uns der Artikel im Dunkeln. Das Rezept: „Es gilt, die Sanktionsfreiheit für das Coaching als sicheren (Zwischen-)Raum für kreative Problemlösungen zu etablieren“, kann wohl nicht die Antwort sein. Denn selbst Organisationen ohne Hierarchien können kaum auf die Möglichkeit von Sanktionen verzichten. Auch dort stellt sich beispielsweise die Frage, wie man mit Mitarbeitenden umgeht, die sich nicht an Regeln halten, eine zu schwache Leistung an den Tag legen, andere Personen schlecht behandeln usw. In hierarchischen Organisationen bleibt es häufig Aufgabe der Führungskraft, sich dieser Fragen anzunehmen. Da hilft auch eine „Doppelausbildung Coaching und Führung“ nicht viel, denn die Rollenkonflikte bleiben bestehen.

Immerhin weisen die Autoren zu Recht auf die Problematik hin, die ich auch früher schon erwähnt habe: Wenn die Führungskräfte qualitativ so gut wie professionelle Coaches ihre Mitarbeitenden beraten sollten, dann müssten sie über eine mindestens so gute Qualifikation verfügen wie Coaches. Aber dann sind sie Coach und keine Führungskraft. Dazu stellen die Autoren fest, ,dass zwar zahlreiche (...) 
kurzzeitige Trainings angeboten werden, die der Komplexität des Beratungsformats jedoch kaum gerecht werden“. Man könnte daraus sogar folgern: Wenn die Autoren schon für ein ,eigenständig zu fundierendes Beratungsformat“ plädieren, dann wäre die Konsequenz daraus, dass es eine Qualifizierung bräuchte, die über ein Doppelformat hinausginge und die Führungskräfte als Coach noch intensiver weiterbilden müssten, da ihre Rolle noch um einiges komplexer ist als die einer/s „,normalen“ professionellen Coachs.

Ein Modul könnte dann z. B. darin bestehen, wie die Führungskraft ihre Mitarbeitenden im Umgang mit der eigenen Führungskraft coacht. Denn wenn ich an meine Führungscoachings denke, dann ist bei fast allen Kund/innen die eigene Führungskraft in mindestens einer Sitzung ein Thema. Besonders eignen würde sich da z. B. die in der Gestalttherapie etablierte „Stuhl-Methode“: Die Führungskraft als Coach bittet ihre/ $n$ Mitarbeitenden, mittels Gestalt-Dialog sich mit ihrer Führungskraft auseinanderzusetzen, wenn es z.B. um das nächste Gehalts-Gespräch geht, oder auch bei anderen Verhandlungen, Konflikten oder weiteren delikaten Themen. Dass ein solches Coaching in der Führungsrolle noch viel anspruchsvoller ist als in der Rolle des unabhängigen Coachs, dürfte dabei deutlich werden.

Das freut dann auch die Weiterbildungsinstitutionen und Anbieter von CoachingQualifikationen, zu denen auch ich mich zähle: Wenn wir flächendeckend alle Führungskräfte zu professionellen Coaches weiterqualifizieren dürfen, dann geht uns so schnell die Arbeit nicht aus. Und wie die Autoren richtig bemerken, braucht es ja dann auch noch supervisorische Begleitung der Führungskräfte, die ihre Mitarbeitenden coachen. Das Schneeballprinzip, von dem Stefan Kühl (2006) geschrieben hat, kommt somit noch ausgeprägter zur Geltung. Aber Supervision wäre durchaus nötig. Denn die Führungskraft als Coach müsste sich auch intensiv mit der Thematik des Loyalitätskonfliktes auseinandersetzen, in denen sie sich befindet: Denn Führungskräfte werden angehalten und auch von der Organisation dafür honoriert, die Interessen und die Ausrichtung der Organisation zu vertreten. Diese Interessen sind nicht zwingend die gleichen wie diejenigen der Mitarbeitenden (Umstrukturierungen, Rationalisierungen usw.). Gleichzeitig erwarten die Mitarbeitenden die Loyalität ihrer Führungskraft und dass sie sich für ihre Interessen einsetzt. Da besteht die Gefahr, dass es zynisch wirken kann, wenn die Führungskraft die Mitarbeitenden coacht beim Erreichen von Zielen, die diese gar nicht von sich aus gewünscht haben.

Dazu kommt noch die Frage, ob denn die Mitarbeitenden überhaupt von ihren Vorgesetzten gecoacht werden wollen. Welche inneren Konflikte entstehen, wenn die Führungskraft die neu erlernten Fähigkeiten unbedingt ausprobieren will und diese nicht möchten? Welche Konsequenzen hat ein Nein, ist in diesem Rahmen eine Freiwilligkeit überhaupt möglich? Wie lange muss ein/e Mitarbeitend/er das Coaching ertragen, wenn er/sie merkt, dass es nicht hilfreich ist? Ein Wechsel des/der Coach ist in diesem Kontext nicht so einfach wie bei einer unabhängigen Person; es kann schnell zu Double-bind-Situationen kommen.

Von daher drängt sich die nächste Frage auf: Wer sagt denn, dass in Organisationen mit flachen Hierarchien nur die Führungskräfte andere Mitarbeitende coachen sollen? Viel naheliegender ist es doch, dass sich Mitarbeitende untereinander coachen. Das geschieht allerdings auch nicht ganz ohne Eigeninteressen, wenn sich z. B. zwei Mitarbeitende um eine gleiche Aufgabe bewerben. Aber zumindest haben Kol- 
leg/innen untereinander keine Sanktionsmacht, hier wäre also die geforderte „Sanktionsfreiheit" bereits Tatsache. Und in diese Richtung soll ja gemäß den Autoren die Entwicklung auch gehen, indem ,noch stärker selbstbestimmte Reflexionsverfahren wie das Selbstcoaching und neue Formen der Intervision an Bedeutung gewinnen werden“.

Da bin ich mit den Autoren einig: Als langjähriger Verfechter von Intervision (Lippmann 2013) vertrete ich die Meinung, dass diese Form der kollegialen Beratung einer Umgangsform auf Augenhöhe sehr gerecht werden kann. Führungskräfte, die selber auch noch operativ tätig sind, können sich darin selber auch engagieren, sowohl als Moderator/in wie auch als Fallgeber/in, und damit eine Kultur auf Augenhöhe tatsächlich fördern, indem sie eben nicht nur „Coach“ sind, sondern sich selber auch von den Mitarbeitenden und/oder Kolleg/innen beraten lassen. Dass dies nicht bei allen Fragestellungen geht, sollte angesichts der Rollenthematik auch klar sein. Und was das Selbstcoaching betrifft: Wenn die Fragen der Übertragung und Gegenübertragung in diesem Setting gut gelöst werden können, wieso nicht auch dieses Format propagieren?

Allerdings plädiere ich als Anbieter sowohl von Führungsentwicklungen wie auch von Coaching-Qualifizierungen auch hier dafür, dass es dazu eine profunde Weiterbildung braucht. Dann haben wir in der Weiterbildung und in der damit verbundenen Supervision noch mehr Kunden, die wir gewinnen können - eine durchaus interessante weitere Geschäftsperspektive ...

\section{Literatur}

Kühl, S. (2006). Die Supervision auf dem Weg zur Profession? Organisationsberatung, Supervision, Coaching, 13(1), 5-18.

Laloux, F. (2015). Reinventing Organizations. Ein Leitfaden zur Gestaltung sinnstiftender Formen der Zusammenarbeit. München: Vahlen.

Lippmann, E. (2013). Intervision. Kollegiales Coaching professionell gestalten. Berlin, Heidelberg: Springer.

Oestereich, B., \& Schröder, C. (2017). Das kollegial geführte Unternehmen. Ideen und Praktiken für die agile Organisation von morgen. München: Vahlen.

Roberston, B. J. (2016). Holacracy. Ein revolutionäres Management-System für eine volatile Welt. München: Vahlen.

Tonhäuser, C. (2010). Implementierung von Coaching als Instrument der Personalentwicklung in deutschen Großunternehmen. Frankfurt/M.: Peter Lang. 